\title{
Investigation of scour onset under seabed pipelines with geometric irregularities
}

\author{
T. Griffiths ${ }^{(1,2)}$, S. Draper ${ }^{(1,2)}$, W. Sun ${ }^{(2)}$, D. White ${ }^{(1,2)}$, L. Cheng ${ }^{(2)} \&$ H. An ${ }^{(2)}$ \\ (1) Centre for Offshore Foundation Systems, University of Western Australia, Perth Australia \\ (2) School of Civil, Environmental and Mining Engineering, University of Western Australia, Perth Australia
}

\begin{abstract}
Based on previous studies, it is well understood that the hydrodynamic pressure difference across the pipeline is responsible for initiation of piping and the onset of scour. However, the influence of geometric irregularities such as field joints on the onset of scour has not previously been investigated. This new study has been stimulated by field observations of subsea pipelines that have indicated that scour holes tend to occur at field joint locations. This paper summarizes a series of experimental results using The University of Western Australia's (UWA)'s unique O-tube test facilities investigating onset of scour underneath scaled model pipelines with a field joint in steady currents. The results of this work are benchmarked against experimental tests and published results for prismatic / uniform pipes. It is demonstrated that periodic geometric irregularities such as field joints have a significant influence on the onset of scour underneath a pipeline.
\end{abstract}

KEY WORDS: Scour, piping, subsea pipeline, field joint, anode, STABLEpipe JIP.

\section{INTRODUCTION}

\subsection{Motivation}

The research reported in this paper forms part of an ongoing body of work into the behavior of subsea pipelines when placed onto erodible seabeds. This research program includes work being undertaken within the STABLEpipe JIP, with sponsorship by Woodside Energy and Chevron and with JIP participation by UWA and Wood Group Kenny. A number of other prominent industry organisations and technical authorities have also contributed to the JIP, whose primary aim is to generate new engineering guidance enabling the effects of mobile and erodible seabeds to be captured in design. The scope of this JIP is described by Griffiths et al. (2010) with the new design paradigms described by Fogliani (2013). The cornerstone of this JIP is physical modelling of the tripartite interactions between pipes, mobile seabed soils and the fluid forcing by waves and currents using the world-unique UWA O-tube test facilities, described in more detail later in this paper.

This research work has been undertaken against an ongoing industry trend for the design and ongoing management of subsea pipelines to become increasingly dependent on accurate prediction of the long-term morphology of the seabed around the pipeline, including embedment due to initial laydown, initiation of spans and subsequent evolution of spans, far-field lowering and/or sedimenta- tion. The sequence and progress of these morphodynamic changes have been described in the literature by Fredsøe et al. (1988), Bruschi et al. (1997) and more recently by Cheng et al. (2009, 2014). These recent studies provide methods to predict longitudinal span growth rates from which the timing of pipeline sinking and/or sagging can be predicted. Leckie et al. (2015) have also observed pipeline lowering in the field, and the apparent dependence of the lowering mechanism on the locations of span initiation along a pipeline (i.e. the prevalence of the pipe (i) sagging into long widely spaced spans, or (ii) sinking into soil shoulders between regular closelyspaced spans). Figure 1, for example, presents an example from actual field observations of a pipeline lowering into the seabed due to sinking.

The relevance of these morphodynamic changes in pipeline condition are typically of first-order importance in predicting the:

- On-bottom stability of the pipeline under large storms (as described by Draper et al., 2015);

- Global buckling response caused by temperature and pressure due to scour-induced modification of the as-laid pipeline embedment (as discussed by Rodriguez et al., 2013);

- Seabed contribution to thermal insulation and thermal inertia relevant to the flow-assurance modelling and management, as described by Zakarian et al. (2012). 

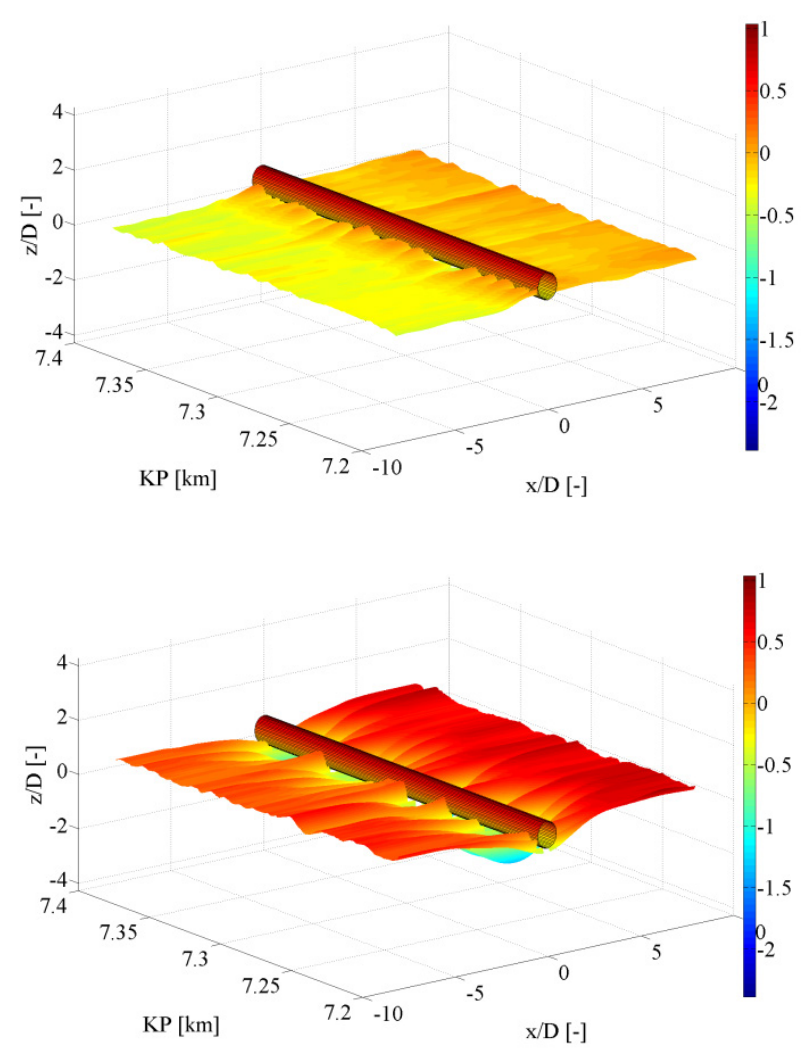

Figure 1 (Top) 6 months after pipelay. (Bottom) 4.5 years after pipelay (Figures reproduced from Leckie et al. 2015)

\subsection{Existing Research into the Initiation of Scour}

The morphology changes described above are predicated on the formation of initial pipeline spans either through the irregular profile of the seabed leading to pipe non-conformity and gaps, or through the initiation of tunnel scour by piping. Extensive prior research has been undertaken into the mechanism and occurrence of piping, which has been well summarized by Sumer et al. (2001). Under steady currents the onset of piping can be predicted using the following empirical formula:

$$
\frac{U_{c r}{ }^{2}}{g D(1-n)(s-1)}=0.025 \exp \left[9\left(\frac{e}{D}\right)^{0.5}\right]
$$

where $U_{\text {cr }}$ is the critical steady current velocity required to cause piping (referenced at the elevation of the top of the pipe); $\mathrm{g}$ is gravity; D is the pipe hydrodynamic diameter; $n$ is the porosity of the sediment; $s$ is the specific gravity of the sediment grains; and $\mathrm{e}$ is the embedment of the pipeline into an otherwise flat seabed. This criteria is applicable at an instant, for a known embedment that is equal on both sides of the pipe.

Further physical modelling of the piping phenomenon has been performed by Zhang et al. (2013) who made two key observations that add the influence of temporal changes in embedment caused by sediment transport:

1. That the onset of piping when the current is progressively increased is influenced by the length of upstream sand-bed available to supply an incoming bedload transport. This length influences the scour / sedimentation budget around the pipeline and hence the propensity for erosion or sedimentation to occur, and therefore for embedment, e, to change;

2. That the duration of the test prior to the onset of piping can have an influence on the velocity at which piping occurs, due to the potential for greater levels of morphology evolution and change in embedment with increasing duration of tests under locally live-bed conditions (i.e. clear-water or far-field live-bed conditions).

\subsection{Field Observations}

As part of ongoing research efforts into improved understanding of pipeline / seabed interaction, a key activity is undertaking detailed analysis of the seabed morphology around existing pipelines. Extensive survey datasets are available for this research, covering a number of pipelines over many years from immediately post-lay. These observations serve to enable ground-truthing of experimental laboratory testing, but also provide opportunities for observation of new and as-yet unexplained phenomena. Leckie et al. (2015) made the interesting observation that for the pipeline analysed in that study, the histogram of span spacing shows a very strong correlation with the pipeline joint spacing (at about $12.2 \mathrm{~m}$ ) as shown in Figure 2. It is understood that the field joint coating on this pipeline has the same thickness (and therefore outer diameter) but is much smoother than the parent pipe coating. Importantly, the short distance between initiation points clearly influenced the mechanism of pipeline lowering for this pipeline (i.e. the pipeline tended to 'sink' rather than 'sag' during lowering; see Figure 1). This suggests that if field joints are a preferential location for onset of scour, sinking is likely to be the dominant mechanism of lowering.

On another pipeline in the same geographic area, the ROV survey footage of the pipeline shortly after lay showed a large number of very short pipeline spans occurring regularly at the field joint locations as shown in Figure 3. In that case, the pipeline field joints had not been infilled and a geometric step $\left(\mathrm{D}_{2} / \mathrm{D}_{1} \approx 0.84\right.$ and $\mathrm{L} / \mathrm{D}_{1} \approx 0.64$; symbols defined on Figure 7) existed between the diameter of the concrete coating and field joint. These observations suggest some geometric feature of the field joint coating system may be contributing to the formation of pipeline spans through piping, triggering the motivation for the experimental study reported in this paper. 


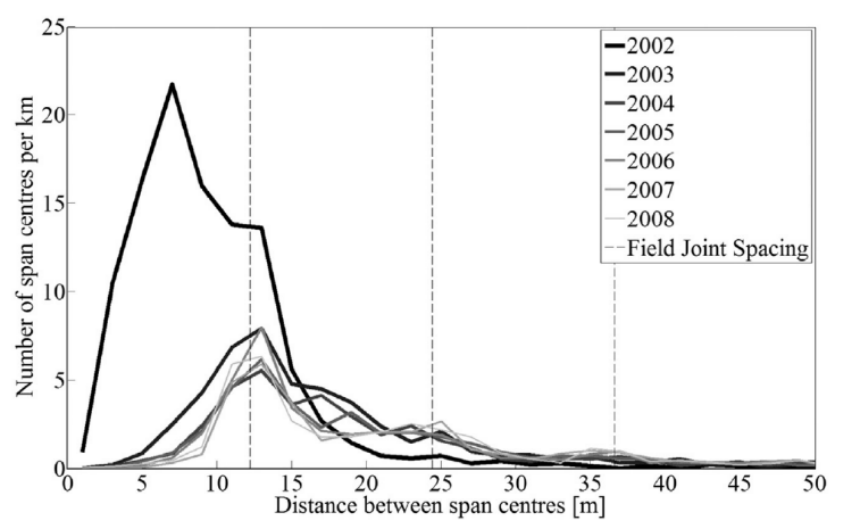

Figure 2 Field Observation of Average Span Spacing (Leckie et al., 2015)

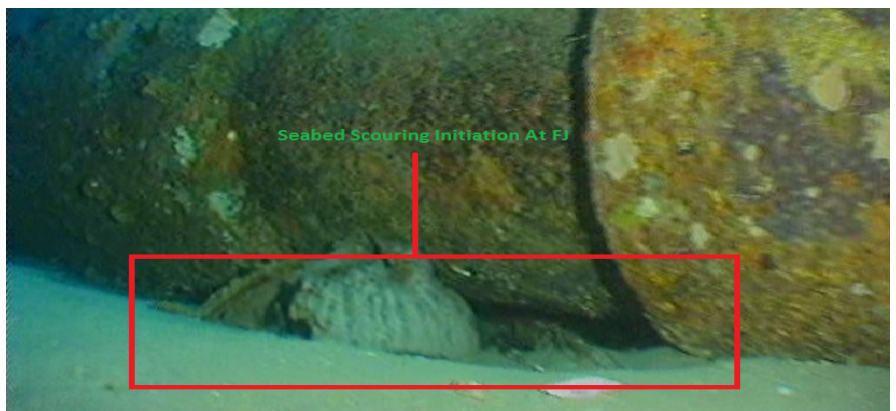

Figure 3 Field Observation of Scour Initiation at a Field Joint Location

\subsection{Geometric Irregularities - Field Joints and Anodes}

The offshore pipeline industry is geared up to handle the manufacture and installation of a variety of different pipeline, umbilical and cable systems. One class of these is rigid steel pipelines which are manufactured in lengths of around $12.2 \mathrm{~m}(40 \mathrm{ft})$, for example as defined in Table 7-19 of DNV-OS-F101 (DNV, 2013). The typical process involves welding together of the joints as shown in Figure 4 followed subsequently by Non-Destructive Testing (NDT) of the welded joints as shown in Figure 5. This requires the concrete, insulation and anti-corrosion coating to be omitted from a short length at the end of each joint, typically around $350 \mathrm{~mm}$ each end, in order to allow sufficient space for the welding 'bug', NDT test head and guide-band(s). As addressed in DNVRP-F102 (DNV, 2011), it is often not a requirement to subsequently infill the gap between the parent concrete coating, resulting in an annular reduction in the diameter of the pipe. This annular reduction has a thickness equal to the thickness of the concrete coating and a typical length of $0.7 \mathrm{~m}$.

For other pipelines, including rigid steel pipelines which are installed by reeling (for example as described by Manouchehri et al., 2008), the process of reeling requires anti-corrosion anodes and other appurtenances to be installed onto the pipe immediately prior to the pipe leaving the vessel. This results in these items representing geometric irregularities lo- cated typically at intervals of less than $300 \mathrm{~m}$, as described by DNV-RP-F103 (DNV, 2010). These irregularities may also act as efficient scour initiators, but are not discussed in detail in this paper.

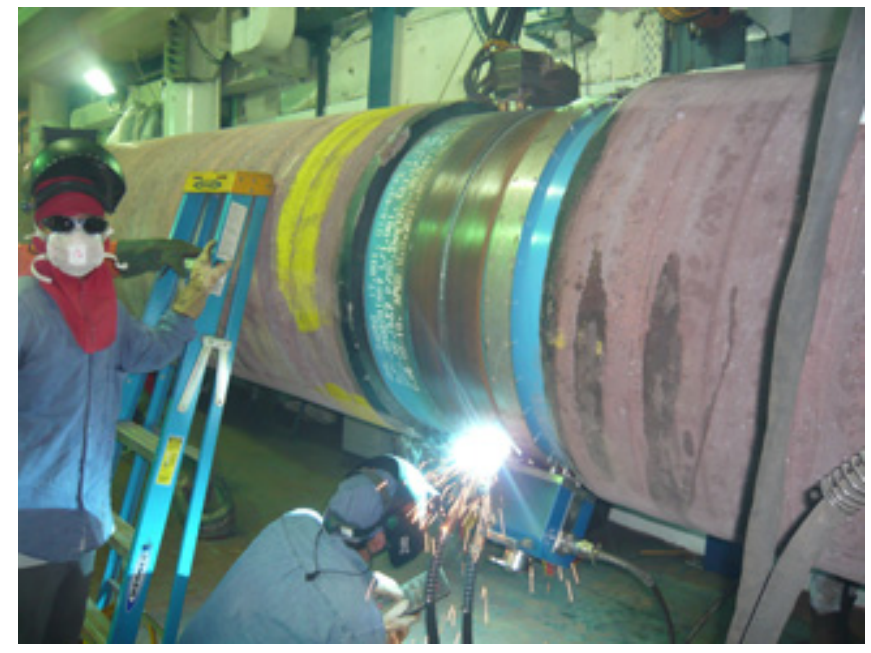

Figure 4 Example of Offshore Pipeline Welding (Lincoln Electric, 2015)

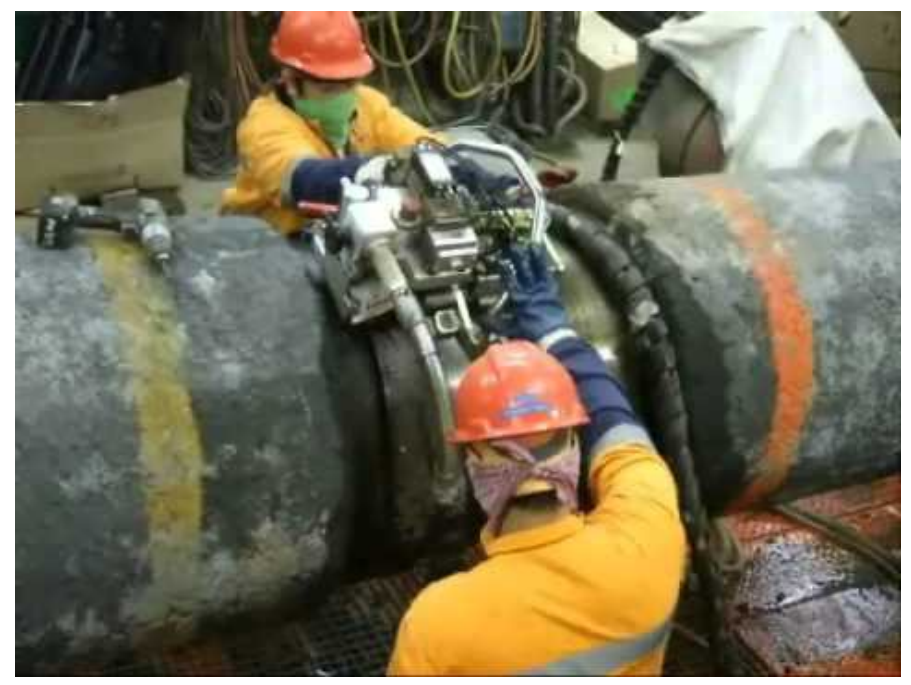

Figure 5 Example of Offshore Pipeline NDT (Rörvik, 2011)

\section{EXPERIMENTAL SETUP}

\subsection{UWA Small O-tube}

The University of Western Australia (UWA) is host to a set of facilities for modelling fluid-structureseabed interaction, based around the innovative Otube technology. Previous publications have described the Large and Mini O-tube test facilities (see An et al. 2013 and Cheng et al. 2014) which are closed-circuit recirculating wave and current flumes, driven by an inline impellor. The O-tube flumes allow seabed flows to be simulated at a range of scales, including full scale modelling of small pipelines under arbitrary combinations of steady currents, regular or irregular wave conditions. Interactions with mobile sediments and infrastructure can be tested and monitored. A new O-tube has recently 
been fabricated and commissioned with dimensions and performance between that of the Large and Mini O-tubes (White et al. 2014). The experimental testing performed for this paper has been undertaken in this new Small O-tube (SOT), which features the key parameters presented in Table 1, with a photograph of the facility shown in Figure 6. As with most recirculating test facilities the uniformity of flow across the test section is not perfect, but the addition of mesh with asymmetric thickness within the flow conditioning section has improved the flow uniformity under steady currents to acceptable levels.

Table 1: Summary of the Small O-tube (SOT) Key Parameters

\begin{tabular}{lcc} 
Dimension & Value & Value \\
\hline Overall length & 6.54 & $\mathrm{~m}$ \\
Overall breadth & 1.85 & $\mathrm{~m}$ \\
Height of test section (in- & 0.45 & $\mathrm{~m}$ \\
cluding soil) & 0.15 & $\mathrm{~m}$ \\
Depth of soil bed & 0.3 & $\mathrm{~m}$ \\
Breadth of test section & 3.0 & $\mathrm{~m}$ \\
Length of test section & 4.5 & $\mathrm{~m} / \mathrm{s}$ \\
Maximum steady bulk ve- & & $\mathrm{m} / \mathrm{s}$ \\
locity & 2.0 & $\mathrm{M}$ \\
ty @ 6 s period & &
\end{tabular}

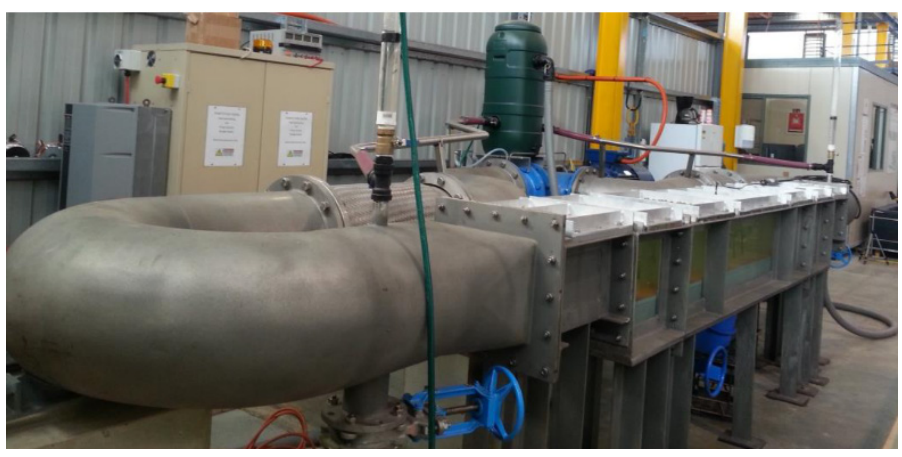

Figure 6: Photograph of UWA Small O-tube (SOT)

\subsection{Model Pipes Including Field Joints}

As noted previously, the geometry of pipeline field joints features a relatively fixed length, $\mathrm{L}$, of $0.7 \mathrm{~m}$, a thickness $\left(D_{1}-D_{2}\right)$ equal to the applied parent pipe coatings, and the diameter of the steel pipeline $\left(D_{2}\right)$, with the notation given in Figure 7 . The model pipes shown in Table 2 were manufactured for this research based on the industry experience of practical limitations on field joint dimensions arising from the range of pipelines and coating thicknesses.
Table 2: The Model Pipe Dimensions

\begin{tabular}{ccccc}
$\mathbf{D}_{\mathbf{2}} / \mathbf{D}_{\mathbf{1}}$ & $\mathbf{L} / \mathbf{D}_{\mathbf{1}}$ & $\mathbf{D}_{\mathbf{1}} \mathbf{( \mathbf { m m } )}$ & $\mathbf{D}_{\mathbf{2}}(\mathbf{m m})$ & $\mathbf{L}(\mathbf{m m})$ \\
\hline $4 / 5$ & 1 & 50 & 40 & 50 \\
$3 / 5$ & $1 / 4$ & 50 & 30 & 12.5 \\
$3 / 5$ & 1 & 50 & 30 & 50 \\
$3 / 5$ & 2 & 50 & 30 & 100 \\
$1 / 1$ & N/A & N/A & 30 & N/A \\
$1 / 1$ & N/A & N/A & 50 & N/A \\
\hline
\end{tabular}

The dimensions are as defined in Figure 7 and a photograph of the model pipes is presented in Figure 8 . The actual model pipe dimensions were selected with due regard for limiting the blockage ratio and length/diameter aspect ratio in the SOT test working section, as well as available plastic bar diameters. The pipes have been designated according to the convention D1-D2-L for identification, for example the first pipe listed in Table 2 is 50-40-50.

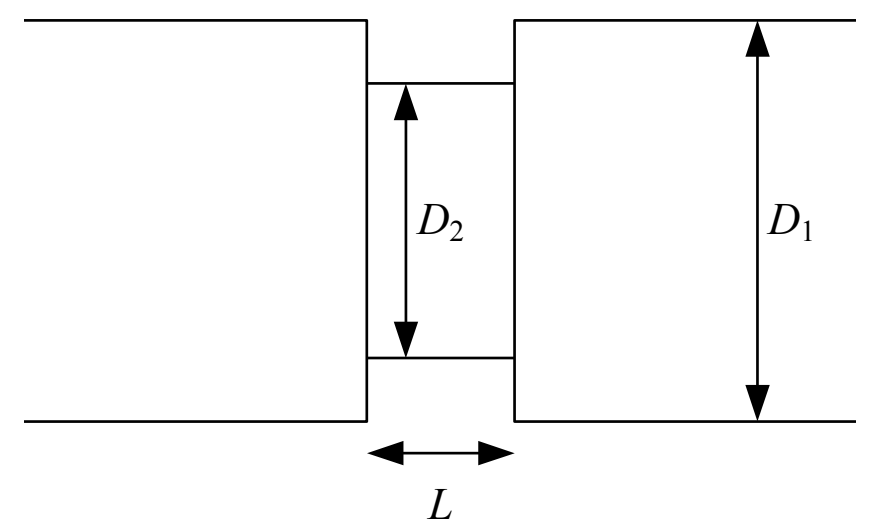

Figure 7: Model Pipe Field Joint and Anode Dimensions

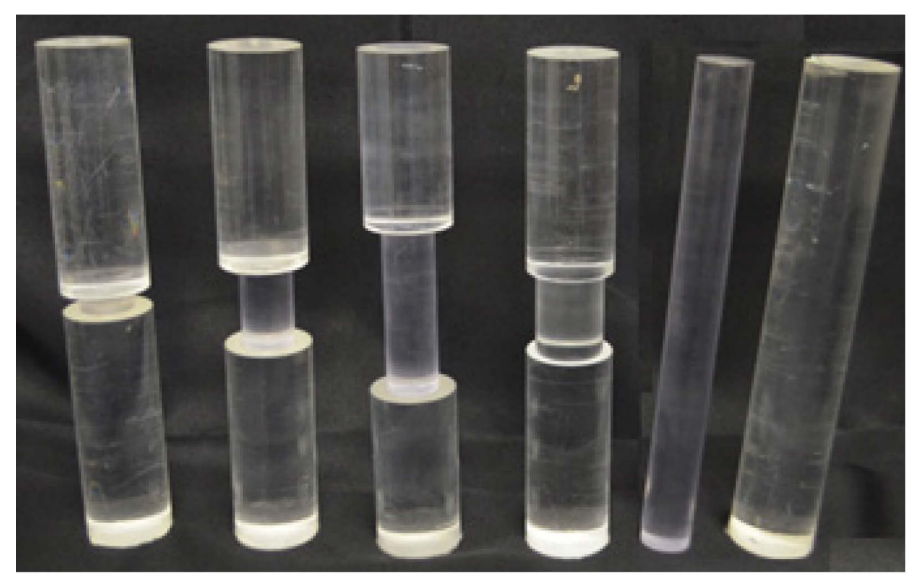

Figure 8 Photograph of Model Field Joints and Regular Pipes

\subsection{Flow Conditions Investigated}

Steady currents were investigated in the experiments, measured using an ADV at the upstream side of the scaled model pipe at the elevation of the top of the model pipe (referenced to the $\mathrm{D}_{2}$ section for the field joint cases). It was found that the steady current velocity in the test section was linearly related to the impellor rotation speed, $\mathrm{N}(\mathrm{u}$ in $\mathrm{m} / \mathrm{s}, \mathrm{N}$ in rpm) 
$u=0.0020 N \quad(N \geq 0)$

The tests were undertaken by rapidly increasing the flow to the 'initial' flow velocity followed by incremental step-increases in velocity typically applied at $5 \mathrm{~s}$ intervals by control of the impellor rpm.

\subsection{Seabed Soil and Embedment Setup}

The sand used was a siliceous sand with a narrow grading and $\mathrm{d}_{50}$ of $0.24 \mathrm{~mm}$. The particle specific gravity was 2.65 , average porosity $40 \%$ and coefficient of uniformity 1.77. The full length of the SOT test section was filled with sand. The closed-loop form of the SOT, therefore, results in continual upstream supply of sediment as sand is lost from the outlet of the test section and returned to the inlet. This results in a richer upstream supply than the alternative of using just a short sand-tray in the vicinity of the model pipe. Experience in the use of this facility has enabled testing practices to be adopted which pre-seed the return pipework with an approximately equilibrium volume of sand, minimizing the variation in inlet sediment supply with flow velocity or time.

The technique for setting up the initial embedment and compaction of sand around the model pipe was refined during the experimental phase to give good repeatability of results. The pipe was setup in the test section and checked for vertical and horizontal alignment normal to the walls, as shown in Figure 9.

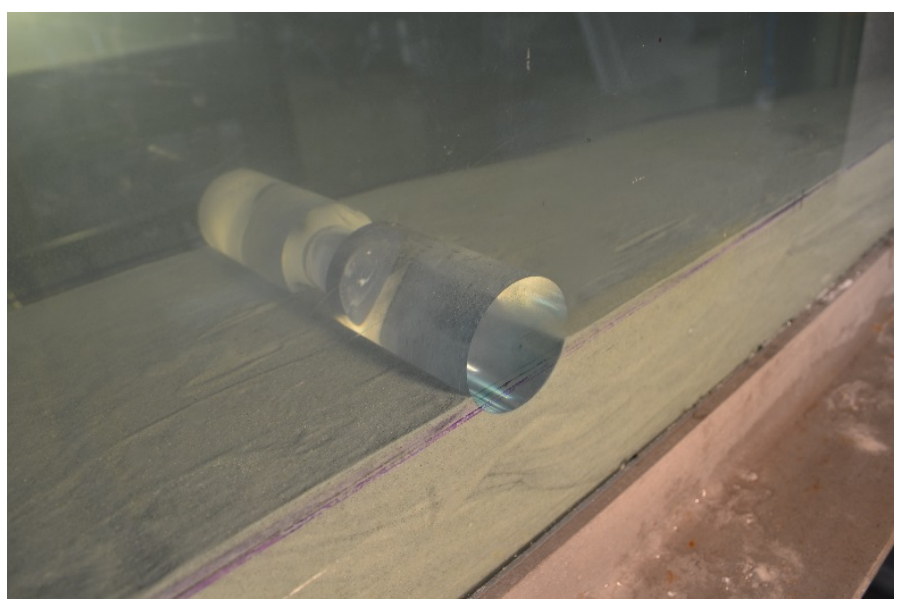

Figure 9: Initial Setup of Pipe in SOT Test Section (50-30-50 Pipe)

The sand was then backfilled around the pipe after each test with tapered plastic 'spatulas' used to compress the sand under the pipe before wiping excess sand away to leave a flat seabed compacted to a consistent density. The model pipe was then photographed end-on and the ratio of $\mathrm{D}_{2}$ to the chord length of the sand-pipe contact measured to calculate the achieved local embedment as shown in Figure 10.

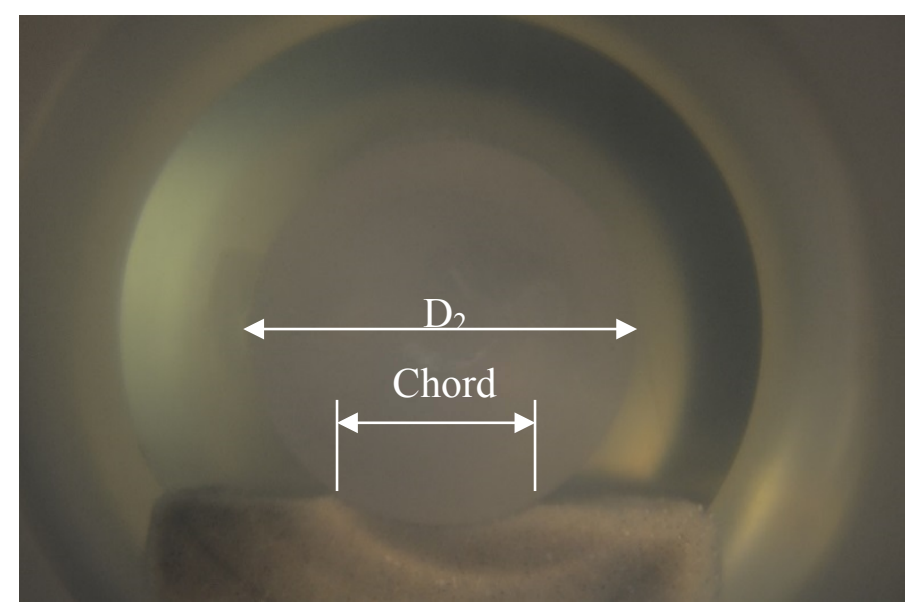

Figure 10: Measurement of Embedment Using Sand Chord (Looking End-on Through 50-30-50 Model Pipe)

This was found to give repeatable results even with embedment as low as $1 \mathrm{~mm}$, with considerably less uncertainty on embedment compared to alternative 'direct' measurement methods of vertical embedment. This technique also ensured the sand was flat and level on both sides of the pipe.

\section{RESULTS}

The results for onset of piping have been normalised using Eq. (1) and are presented in Figure 12. These results are discussed in more detail below, but have omitted cases where the time to reach piping resulted in excessive sedimentation to occur around the pipe, particularly on the lee-side, meaning that the initial embedment did not represent the conditions at onset. This effect is discussed further below.

\subsection{Effect of Sedimentation on Results}

As noted by Zhang et al. (2013), the occurrence of sedimentation against the pipe (especially on the lee side) leads to an increase in the seepage path-length for the flow and therefore an increase in the effective embedment for triggering piping and causing the onset of scour. Such behaviour was certainly observed in this test programme, with several cases resulting in complete burial of the model pipe as shown in Figure 11. 


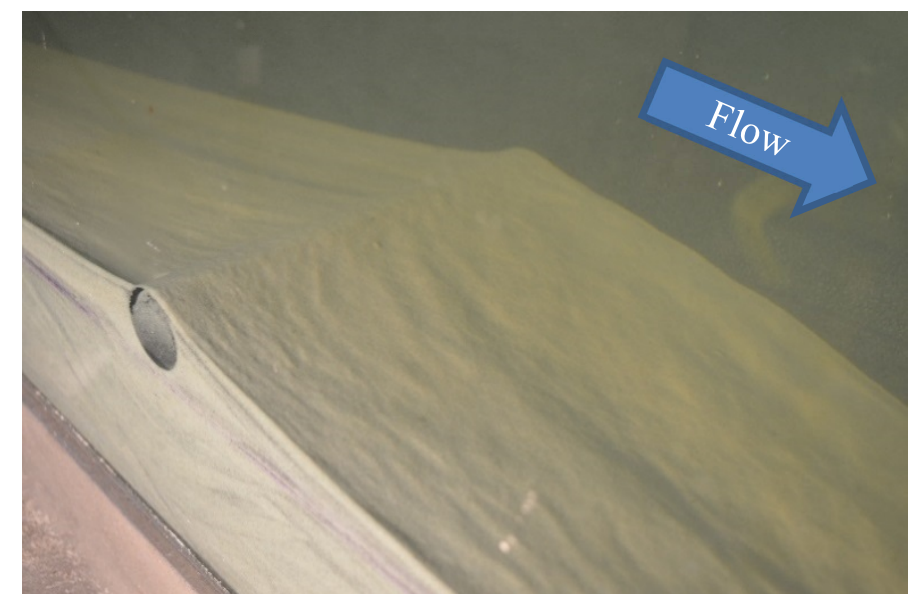

Figure 11 Effect of Sedimentation on Onset of Scour
The rate at which this sedimentation occurs increases as the flow velocity increases above the local sediment mobility threshold, while the progress of sedimentation towards an equilibrium profile increases with both the sedimentation rate and as the flow duration increases. It is considered likely that with further investigation (Griffiths et al., 2016), valuable results can be obtained from those tests where sedimentation resulted in the seabed profile becoming non-flat. However since the primary aim of the tests presented in this work is to investigate the relative influence of geometric irregularities, for the purposes of this work test results where sedimentation resulted in burial of the pipe and suppression of scour were omitted. Consequently, the maximum test duration included is between $60-80 \mathrm{~s}$ (with $80 \mathrm{~s}$ being for a low-velocity test where sedimentation rate was low).

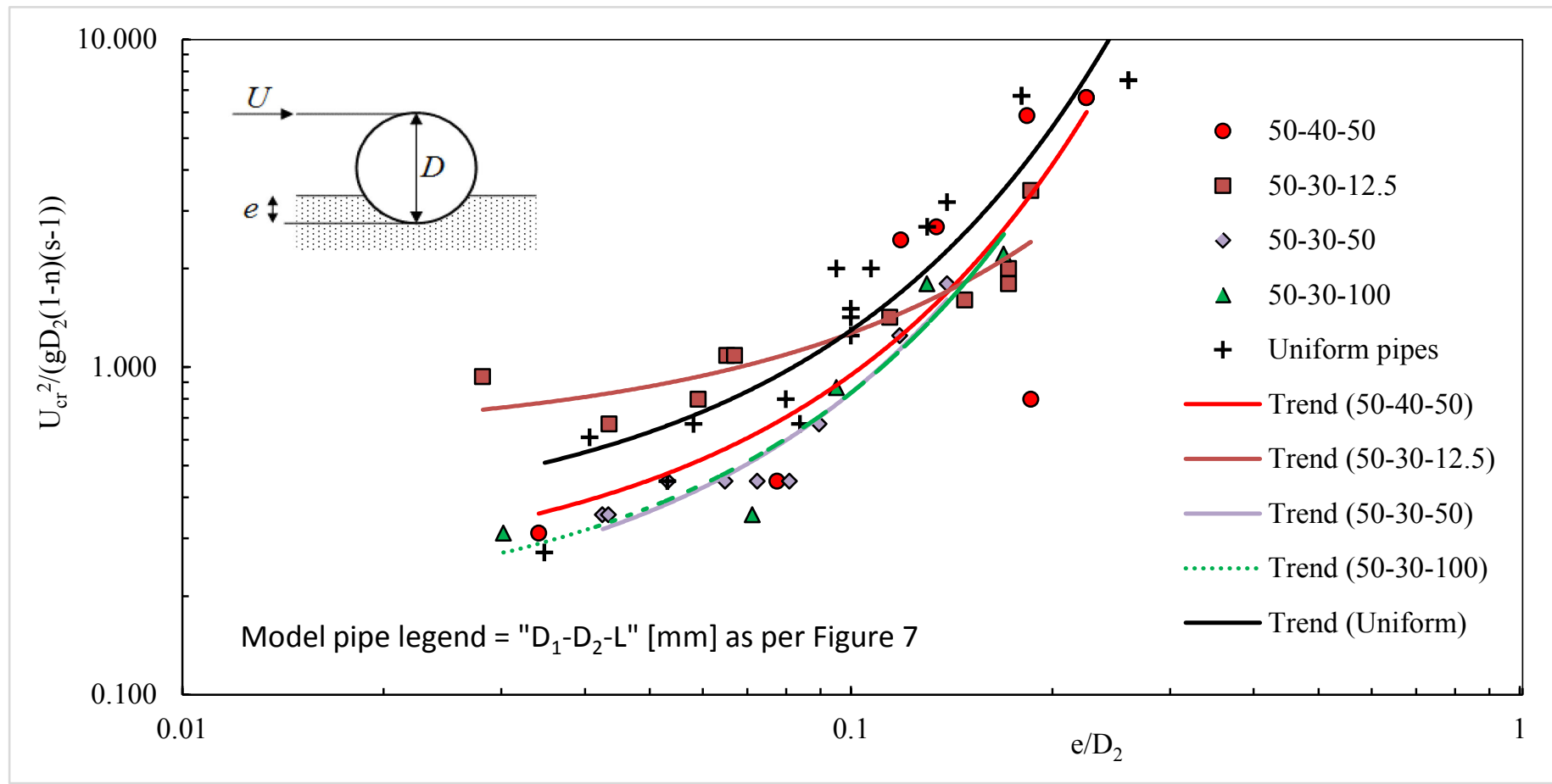

Figure 12: Graphical Summary of Results (Trend lines shown for each pipeline, with colour corresponding to the relevant markers)

\subsection{Comparison with Previously Published Results}

The plain $30 \mathrm{~mm}$ and $50 \mathrm{~mm}$ pipe scour onset results are compared to the results by Sumer et al. (2001) in Figure 13. The observations from this comparison are that:

- The results from this research work show broadly the same exponential form of relationship published by Sumer and Fredsøe (2002), although it is noted our results better fit the parameters given in Eq. (3) with an $\mathrm{R}^{2}$ value of 0.93 . As is discussed further in Griffiths et al. (2016), this best-fit relationship reflects some influence of sedimentation on scour onset;

$$
\frac{U_{c r}{ }^{2}}{g D(1-n)(s-1)}=1.07 \times 10^{-4} \exp \left[14.5\left(\frac{e}{D}\right)^{0.18}\right]
$$

- In the present results a higher flow velocity is required for piping compared to Sumer et al. (2001). The reason for this is likely to be because the present experiments were conducted at a blockage ratio (pipe diameter to flume depth) of between $1 / 10$ and $1 / 6$, whilst the experiments reported in Sumer and Fredsøe (2002) were performed mostly at a blockage of $1 / 3$. Zang et al. (2009) has shown that blockage al- 
ters the pressure difference either side of the pipeline and the potential for onset of scour due to piping. The prediction of critical conditions for onset of scour due to Zang et al. (2009) at a blockage of $1 / 10$ has been found by curvefitting to be given by Eq (4) and also shown in Figure 13. It is found to agree reasonably well with the present experimental results. This observation suggests that Eq (1) will under-predict the velocity at which piping will occur for free field (zero blockage effect) conditions. This bias is unconservative if piping is relied on in design to initiate span development and lowering, hence the Zang et al. (2013) relationship is preferable.

$\frac{U_{c r}{ }^{2}}{g D(1-n)(s-1)}=0.006 \exp \left[7.5\left(\frac{e}{D}\right)^{0.18}\right]$

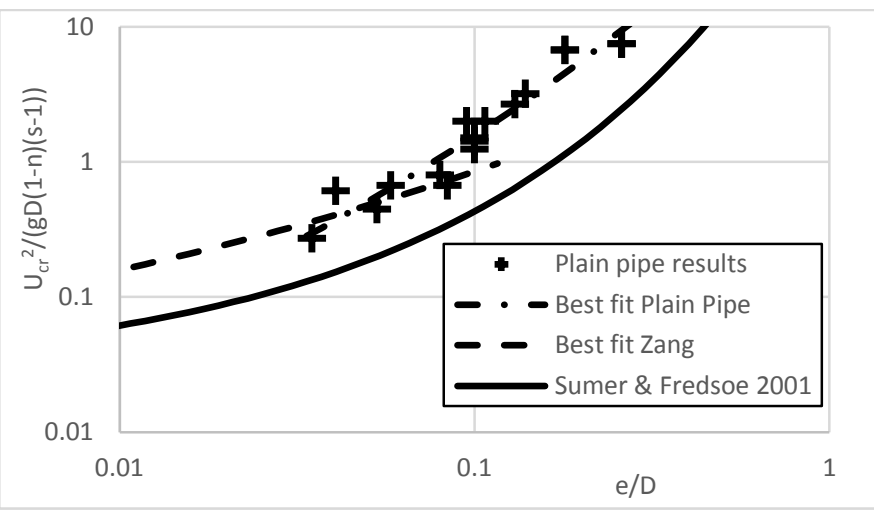

Figure 13 Comparison of Plain Pipe Results With Sumer et al. (2001) and Zang et al. (2009) For Blockage 1/10

\subsection{Effect of Field Joint Gap on Piping Onset}

By using the plain pipe best-fit curve in Eq (3) to normalize the scour onset results as per Eq (5), the normalized effect $(\mathrm{F})$ of different field joint lengths is compared in Figure 14 according to:

$$
F=\frac{\frac{U_{c r}{ }^{2}}{g D(1-n)(s-1)}}{1.07 \times 10^{-4} \exp \left[14.5\left(\frac{e}{D}\right)^{0.18}\right]}
$$

Observations from these results are that:

- The $L / D_{1}=1$ and $=2$ results are very consistent and show a significant decrease compared to plain pipe in the required non-dimensional velocity for onset of piping, with relatively little dependence on the pipe embedment. Piping will occur at a lower velocity at a field joint compared to a uniform pipe with the same diameter as the field joint, for this range of $L / D_{1}$.

- The $L / D_{1}=0.25$ results show a distinctly different relationship which is dependent on the embedment, with the results for shallow embedment showing a lower propensity for onset of scour than for a plain pipe.

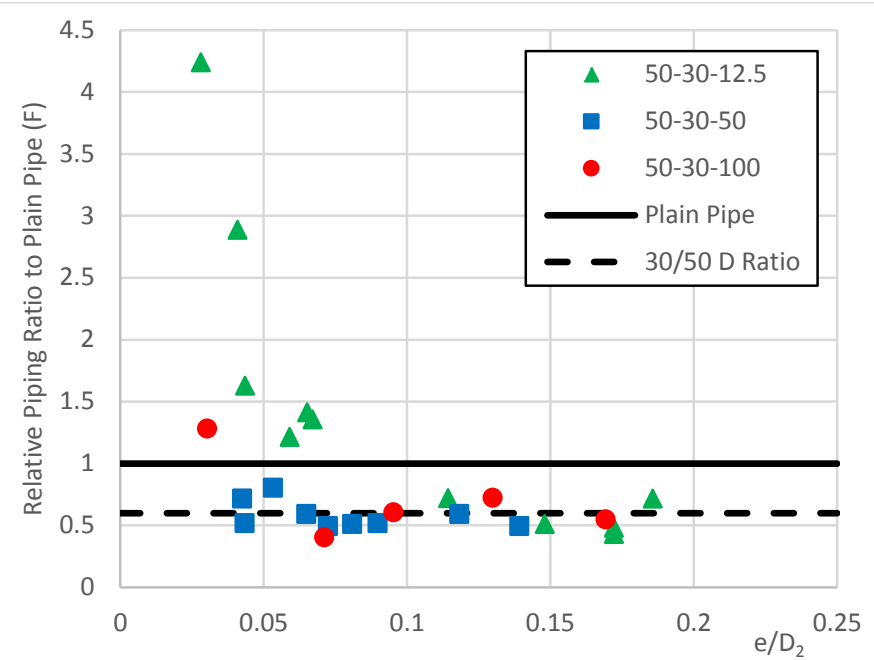

Figure 14 Comparison of Gap Length

The results for $L / D_{1}=1$ and $=2$ can be understood based on the relationship presented in Eq (1) and the derivation of that relationship by Sumer et al. (2001) as the balance between the pressure difference across the pipe over the seepage path-length through the soil. If the pressure difference across the pipeline is dominated by the parent pipe of the larger diameter $\left(D_{1}\right)$ which is many times longer than the field joint, then it might be assumed that this pressure difference from the larger pipe also applies in the corner of the small $\left(\mathrm{D}_{2}\right)$ pipe, however the seepage path-length is only that associated with the smaller pipe. The onset of scour might therefore be expected to occur in the corner of the field joint and at a critical velocity lower than the parent pipe by the ratio of their diameters, as indicated by the dashed line on Figure 14.

The flow behavior during these tests revealed a number of features of interest. These include that the initiation of piping occurred consistently in the corner of the field joint against the step-change in pipe diameter, as shown in Figure 15.

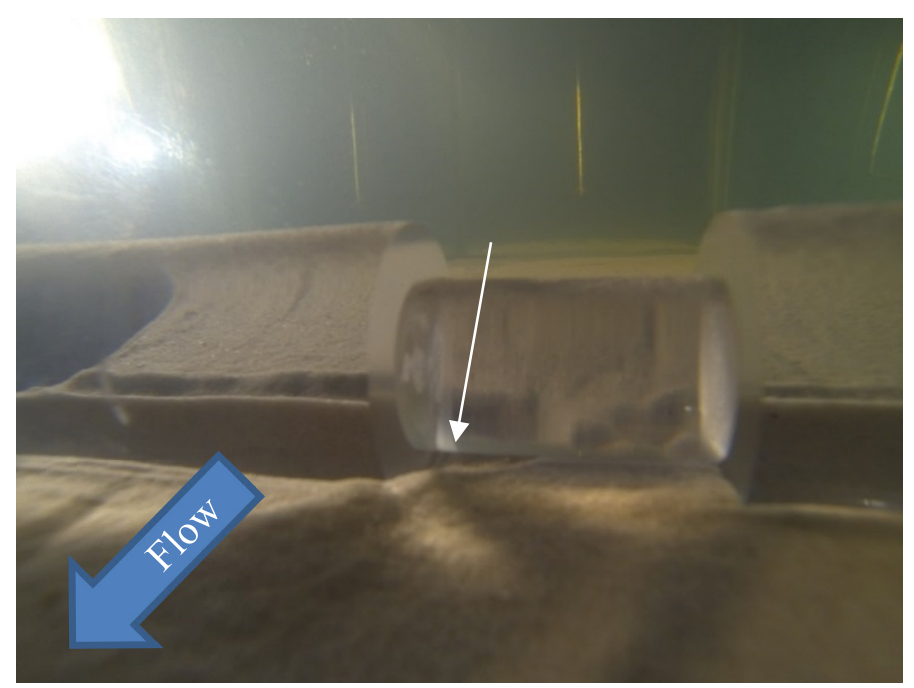

Figure 15 Initiation of Piping in Corner of Field Joint 
It was also consistently observed that horseshoe vortices formed in each corner of the field joint for $L / D_{1}$ $=1$ and $=2$, with the direction of rotation as shown in Figure 16 being opposed to the orientation of the near-bed horizontal vortex upstream of the pipe. In contrast the $\mathrm{L} / \mathrm{D}_{1}=0.25$ gap was only large enough for one vortex to form, with the direction of rotation observed to swap during a test as shown in Figure 17 followed by Figure 18 four seconds later.

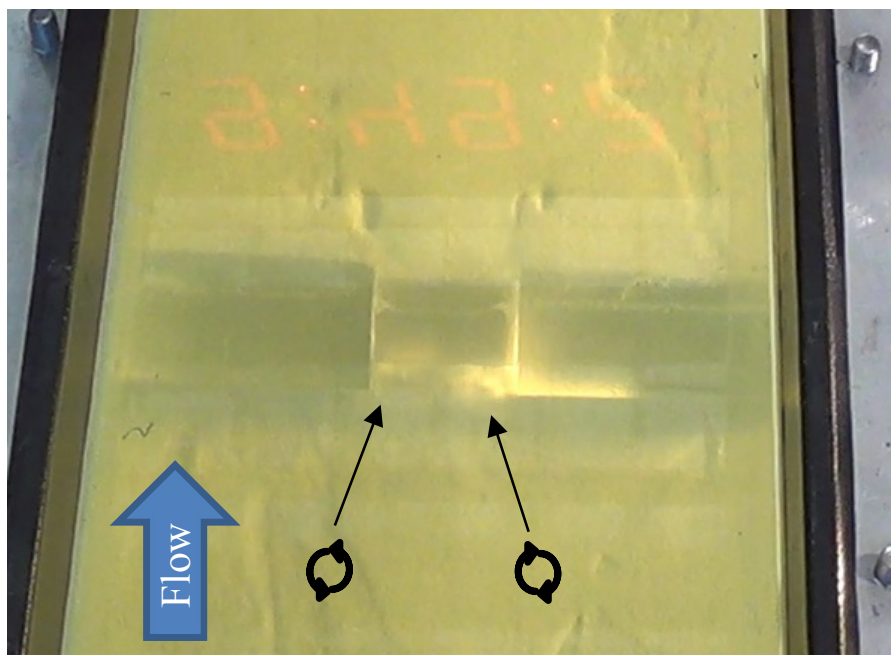

Figure 16 Horseshoe Vortices Formed in Corners

The importance in scour mechanisms of horseshoe vortices formed at the boundary between vertical piles and the seabed has previously been described (See for example Sumer and Fredsøe, 2002, Zhao et al., 2010). The slightly more bathymetrically complex interface between a horizontal seabed pipeline and the side walls of a test flume has been investigated by Ni (2013) as shown in Figure 19. This study found that horseshoe vortices form and are important in understanding the scour morphology and hydrodynamics observed in the UWA O-tubes, where piping consistently occurs inboard of the near-wall horseshoe vortex.

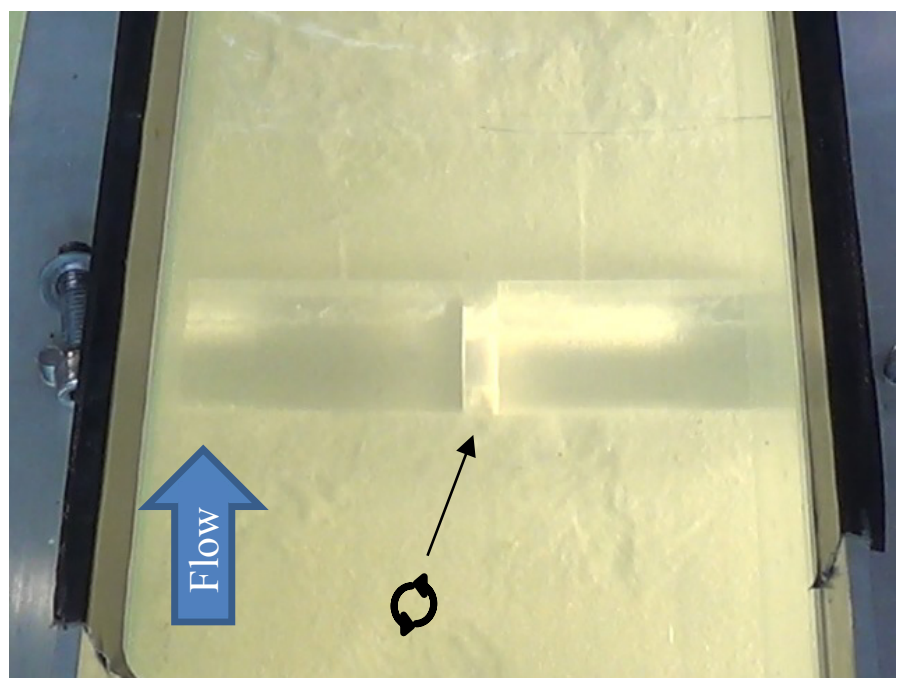

Figure 17 Single Anti-Clockwise Horseshoe Vortex in Gap (94s)

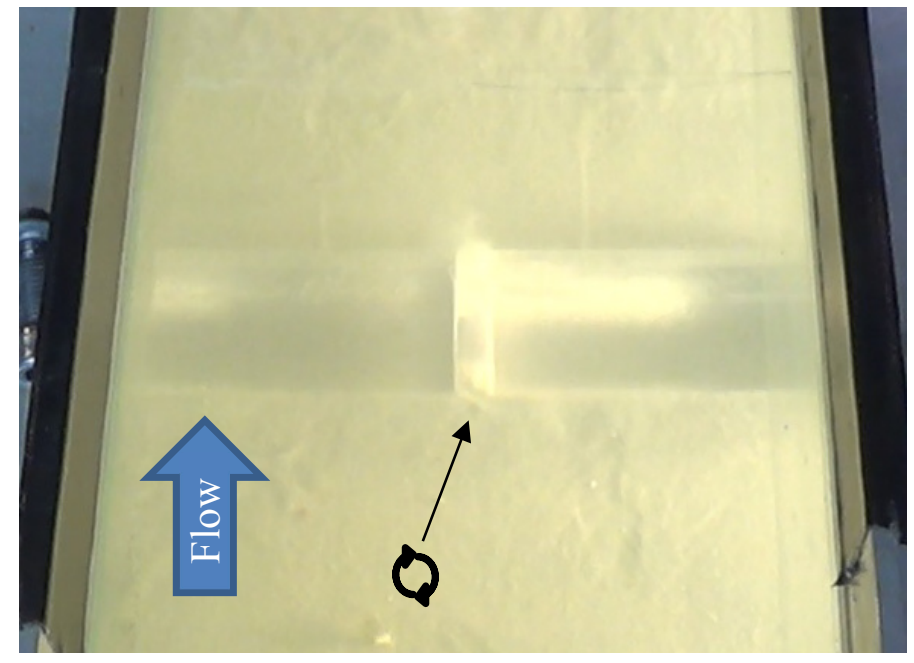

Figure 18 Vortex Flipped to Clockwise (98s)

Figure 19 is also relevant to this field joint testing because of the apparent importance of the horseshoe vortices in sediment transport along the upstream toe of the pipe. Sediment is carried within the upstream horizontal vortex towards the vertical horseshoe vortex, leading to advection of sediment over the pipe. The horseshoe vortices also appear to have an influence on the upstream pressure at the base of the pipe, as evidenced by the test results for $L / D_{1}=0.25$ for shallow embedments. In the absence of some change in the flow and therefore pressures around the field joint pipe, piping would be expected to occur at an upper ratio of $F=1.0$. The dependence of embedment on this behaviour implies an as-yet poorly understood interplay between the change in pressure across the pipe and the sediment supply budget in the field joint gap.

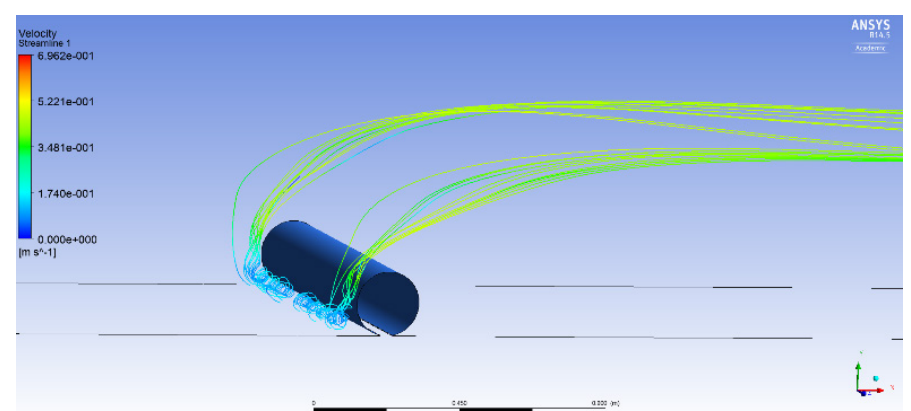

Figure 19 Horseshoe Vortices Formed Against Side-Walls (Ni, 2013)

\subsection{Effect of Field Joint Thickness on Piping Onset}

Again using $\mathrm{Eq}(5)$ to normalize the results compared to plain pipe, the effect of different field joint thicknesses is compared in Figure 20. Observations from these results are that:

- The $\mathrm{D}_{2} / \mathrm{D}_{1}=3 / 5$ results show a much greater reduction in required onset velocity for piping compared to the $D_{2} / D_{1}=4 / 5$ results, with both showing some dependence on embedment;

- The $\mathrm{D}_{2} / \mathrm{D}_{1}=3 / 5$ results show a much lower scatter in results compared to the $\mathrm{D}_{2} / \mathrm{D}_{1}=4 / 5$ results. 


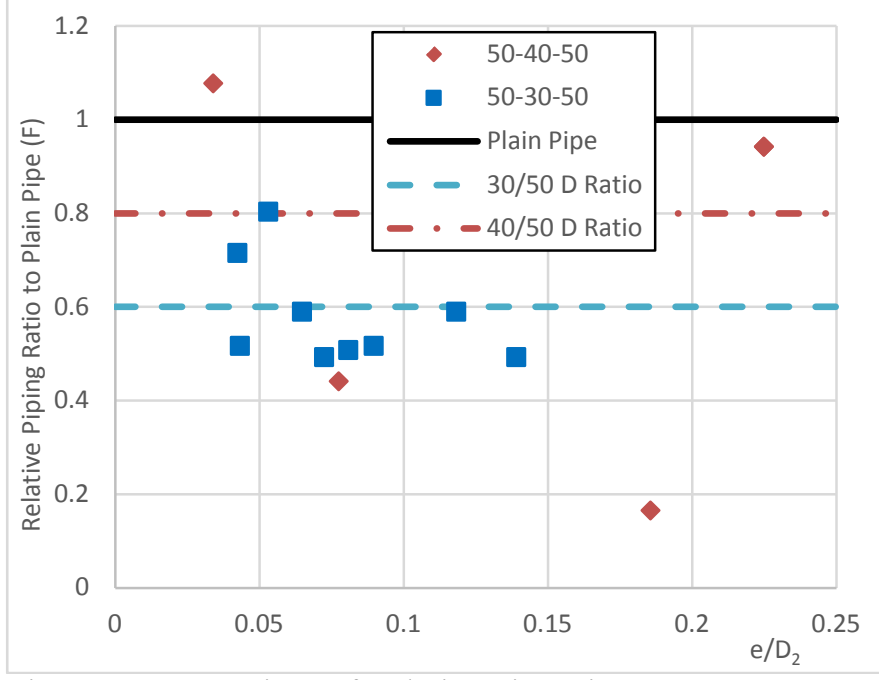

Figure 20 Comparison of Relative Pipe Diameters

The reduction in required velocity for onset of piping as $\mathrm{D}_{2} / \mathrm{D}_{1}$ reduces is an intuitively sensible result, albeit the scatter in the results for 50-40-50 pipe mean that it is difficult to determine how well they fit the piping onset velocity normalized by the 0.8 ratio of pipe diameters. The increasing fluid drag force and pressure difference across the larger diameter pipeline would be expected to drive an increasingly strong horseshoe vortex and pressure differential across the smaller pipe at the shoulder.

The observation from Leckie et al. (2015) that the occurrence of spans is strongly correlated with pipe joint length even when the $D_{2} / D_{1}$ ratio $\approx 1$ but the field joint coating is of significantly different roughness is of great interest. The implication is that it is still easier to initiate scour at these locations, with the exact cause not yet confirmed. It may be that the smooth field joint coating allows enhanced seepage flow against the pipe due to locally higher permeability at the interface, or that drag forces over the field joint are different, hence pressure differentials exist between the field joint and parent pipe lateral locations which are sufficient to trigger early onset of scour.

\section{CONCLUSIONS}

The results of this work show that:

- The presence of geometric irregularities along subsea pipelines have an important influence on the propensity for piping to occur;

- Irrespective of the presence of geometric irregularities, when flow conditions and sufficient upstream supply of sediment allow, significant sedimentation or burial of the pipeline can occur without scour. Further work is required to better interpret the changing propensity for scour initiation with sedimentation;

- Where the aspect ratio of the geometric irregularity is sufficiently large to allow the formation of independent horseshoe vortices in each corner of the step change in diameter, the length of the feature does not appear to influence the propensity for piping to occur;

- Increasing relative diameter of the geometric feature to the main pipe influences the propensity for piping to occur more easily than predicted based on the diameter of the geometric feature, however normalising the pressure difference by the ratio of pipeline diameters appears to be a plausible method;

- However field observation suggests that a change in diameter may not be essential, and that a change in pipe roughness may be sufficient to trigger piping more easily at specific locations than for a plain pipe.

This study highlights that accurate predictions of pipeline scour and self-burial may require field joints and other appurtenances to be considered, since they may control the onset of piping. Also, it indicates that self-burial - and the resulting stabilisation - could be accelerated by deliberate inclusions of surface irregularities on a pipeline during construction. This approach could be a more efficient method of achieving stability compared to conventional primary and secondary stabilisation solutions.

\section{ACKNOWLEDGEMENTS}

The authors gratefully acknowledge the generous support of the STABLEpipe JIP sponsors, Woodside and Chevron, for their support of the UWA O-tube research program. The fourth author acknowledges the support of Shell Australia via the Shell EMI Chair in Offshore Engineering at UWA.

\section{REFERENCES}

An, H., Luo, C., Cheng, L., \& White, D. (2013). A new facility for studying ocean-structure-seabed interactions: The Otube. Coastal Engineering, 82, 88-101.

Bruschi, R., Drago, M., Venturi, M., Jiao, G., \& Sotberg, T. (1997). Pipeline Reliability Across Erodible/Active Seabeds. In Offshore Technology Conference.

Cheng, L., An, H., Luo, C., Brown, T., Draper, S., \& White, D. J. (2014). UWA's O-tube facilities: Physical modelling of fluid-structure-seabed interaction. In ICPMG2014-Physical Modelling in Geotechnics: Proceedings of the 8th International Conference on Physical Modelling in Geotechnics 2014 (ICPMG2014), Perth, Australia, 14-17 January 2014 (p. 3). CRC Press.

Det Norske Veritas (2013), Offshore Standard DNV-OS-F101 Submarine Pipeline Systems.

Det Norske Veritas (2011), Recommended Practice DNV-RPF102 Pipeline Field Joint Coating and Field Repair of Linepipe Coating.

Det Norske Veritas (2010), Recommended Practice DNV-RPF103 Cathodic Protection of Submarine Pipelines by Galvanic Anodes. 
Draper, S., An, H., Cheng, L., White, D. J., \& Griffiths, T. (2015). Stability of subsea pipelines during large storms. Philosophical Transactions of the Royal Society of London A: Mathematical, Physical and Engineering Sciences, 373(2033), 20140106.

Fogliani, N. (2013). Australian Sector Review: STABLEpipe JIP Progress Towards Improved Understanding of Pipeline Stability. In Proceedings of the Offshore Pipeline Technology Conference, Amsterdam.

Rörvik, G. (2011). Gjoa export pipeline installation https://i.ytimg.com/vi/K9M7BKkHnTE/hqdefault.jpg and https://www.youtube.com/watch? $\mathrm{v}=\mathrm{K} 9 \mathrm{M} 7 \mathrm{BKkHnTE}$

Griffiths, T. J., White, D. J., \& Cheng, L. (2010). Progress in Investigating Pipe-Soil-Fluid Interaction: The STABLEpipe JIP. In The Twentieth International Offshore and Polar Engineering Conference. International Society of Offshore and Polar Engineers.

Griffiths, T. J., Draper, D., Sun, W., Cheng, L., An, H. \& White, D. J. (2016). Exploring the bifurcation between sedimentation versus scour onset below pipelines in unidirectional currents. In: Scour and Erosion, Proceedings Eighth International Conference on Scour and Erosion, Oxford, United Kingdom, 12 - 15 September 2016, (in pub.).

Leckie, S. H., Draper, S., White, D. J., Cheng, L., \& Fogliani, A. (2015). Lifelong embedment and spanning of a pipeline on a mobile seabed. Coastal Engineering, 95, 130-146.

Lincoln Electric (2015) http://www.lincolnelectric.com/enus/educationcenter/PublishingImages/ArticleImages/P1100830.JPG

$\mathrm{Ni}$, D. (2013). 3D CFD investigation of the Large O-Tube facility, Final year CEED thesis completed for the School of Civil \& Resource Engineering, The University of Western Australia.

Rodriguez, A B., Bransby, M F., Finnie, I., Low, H E. \& White, D J. (2013). Changes in Pipeline Embedment Due to Sediment Mobility: Observations and Implications for Design. OMAE2013-11425

Sumer, B. M., Truelsen, C., Sichmann, T., \& Fredsøe, J. (2001) Onset of scour below pipelines and self-burial. Coastal engineering 42(4): 313-335.

Sumer, B. M., \& Fredsøe, J. (2002). The mechanics of scour in the marine environment. World Scientific.

White, D.J., Draper, S., Cheng, L., An, H., Mohr, H. \& Leckie, S. (2014), 'Ocean-Structure-Seabed interaction: O-tube modelling of pipeline stability', Australian Geomechanics, 49(4), 157-164.

Zakarian, E., Holbeach, J., \& Morgan, J. E. P. (2012). A holistic approach to steady-state heat transfer from partially and fully buried pipelines. In Offshore Technology Conference. Offshore Technology Conference.

Zang, Z., Cheng, L., Zhao, M., Liang, D. \& Teng, B. (2009). A numerical model for onset of scour below offshore pipelines. Coastal Engineering, 56(4): 458-466.

Zhang, Q., Draper, S., Cheng, L., An, H., \& Shi, H. (2013). Revisiting Mechanics of Onset of Scour Below Pipelines in Steady Current. In Proceedings of International Conference on Ocean, Offshore and Arctic Engineering, OMAE201311334.

Zhao, M., Cheng, L., \& Zang, Z. (2010). Experimental and numerical investigation of local scour around a submerged vertical circular cylinder in steady currents. Coastal Engineering, 57(8), 709-721. 